УДК 661.56+661.98;54.01+54.03

\title{
В.И. Созонтов ${ }^{a}$, А.Г. Архипов ${ }^{a}$, А.В. Суворин ${ }^{a}$, В.М. Москалик ${ }^{a}$, И.Л. Коваленко ${ }^{b}$
}

\section{ИССЛЕДОВАНИЕ СОСТАВА ПАРОВОЙ ФАЗЫ НАД РАССЛАИВАЮЩИМИСЯ ВОДНЫМИ РАСТВОРАМИ АЗОТНОЙ КИСЛОТЫ И ОКСИДОВ АЗОТА}

а Восточноукраинский национальный университет им. В. Даля, г. Северодонецк, ' ГВУЗ «Украинский государственный химико-технологический университет», г. Днепр

\begin{abstract}
В работе теоретически обоснованы и экспериментально изучены закономерности фазового равновесия жидкость-пар в многокомпонентной расслаивающейся системе «оксиды азота-азотная кислота-вода» под давлением 0,1-1,1 МПа. Разработана методика расчета состава паровой фазы, которая учитывает процессы полимеризации диоксида азота и образования оксида азота(III). По значениям парциальных давлений $\mathrm{NO}$ и $\mathrm{NO}_{2}$ рассчитана частная константа равновесия реакции кислотообразования и показано, что для исследованной области массовых соотношений $\mathrm{N}_{2} \mathrm{O}_{4}: \mathrm{H}_{2} \mathrm{O}=1: 2 \ldots 2: 1$ при начальных концентрациях азотной кислоты 40,0-68,4 мас.\% зависимость логарифма частной константы равновесия от равновесной концентрации $\mathrm{HNO}_{3}$ имеет линейный характер, свидетельствующий о независимости частной константы равновесия от давления. Установлено, что частная константа равновесия может быть определена через общую константу равновесия, рассчитанную по термодинамическим константам, и частную константу равновесия, найденную по парциальным давлениям $\mathrm{HNO}_{3}$ и $\mathrm{H}_{2} \mathrm{O}$. Рассчитан состав паровой фазы и показано, что экспериментальные данные удовлетворительно согласуются с данными, полученными по термодинамическим функциям. Относительная ошибка между опытными и расчетными данными составила около 5\%.
\end{abstract}

Ключевые слова: оксиды азота, азотная кислота, равновесный состав, парциальное давление, частная константа равновесия.

DOI: $10.32434 / 0321-4095-2019-123-2-121-126$

\section{Введение}

Одним из наиболее рациональных путей получения растворов $\mathrm{N}_{2} \mathrm{O}_{3}-\mathrm{N}_{2} \mathrm{O}_{4}$ является разложение $\mathrm{N}_{2} \mathrm{O}_{4}$ водой с последующей ректификацией образуюшейся смеси. Основой для создания и осуществления высокоэффективного технологического процесса служат данные по фазовому равновесию жидкость-пар в многокомпонентной системе оксиды азота-азотная кислота-вода.

Вопросу изучения равновесия между оксидами азота и водными растворами азотной кислоты посвящен целый ряд фундаментальных работ [1-5], послуживших основой для изучения процессов абсорбции и десорбции оксидов азота, а также создания крупнотоннажных производств азотной кислоты [6-11]. Однако анализ этих работ показывает, что почти все исследователи изучали равновесие при не слишком высоких парциальных давлениях оксидов азота (как правило, не более 80 кПа) и совершенно не касались изучения равновесия в расслаивающихся системах.

Основная реакция, определяющая процесс кислотообразования, выражается известным уравнением [12]:

$$
\begin{aligned}
& 3 \mathrm{NO}_{2}+\mathrm{H}_{2} \mathrm{O}=2 \mathrm{HNO}_{3}+\mathrm{NO}+17,3 \text { кДж } \\
& \left(\Delta \mathrm{G}_{298}^{0}=2,0 \text { кДж }\right) .
\end{aligned}
$$

Константу равновесия этой реакции принято рассматривать, как произведение двух частных констант:

$$
\mathrm{K}=\frac{\mathrm{P}_{\mathrm{NO}} \cdot \mathrm{P}_{\mathrm{HNO}_{3}}^{2}}{\mathrm{P}_{\mathrm{NO}_{2}}^{3} \cdot \mathrm{P}_{\mathrm{H}_{2} \mathrm{O}}} ; \mathrm{K}=\mathrm{K}_{1} \cdot \mathrm{K}_{2},
$$


где $\mathrm{K}_{1}=\frac{\mathrm{P}_{\mathrm{NO}}}{\mathrm{P}_{\mathrm{NO}_{2}}^{3}} ; \mathrm{K}_{2}=\frac{\mathrm{P}_{\mathrm{HNO}_{3}}^{2}}{\mathrm{P}_{\mathrm{H}_{2} \mathrm{O}}}$.

Величину частной константы равновесия $\mathrm{K}_{2}$, зависящую как от температуры, так и от концентрации азотной кислоты, определяют на основании данных о составе паров над растворами $\mathrm{HNO}_{3}$. Частную константу $\mathrm{K}_{1}$, которая характеризует парциальное давление $\mathrm{NO}$ и $\mathrm{NO}_{2}$ над водными растворами азотной кислоты и оксидов азота, все исследователи определяли экспериментально. Однако, частную константу равновесия $\mathrm{K}_{1}$ с достаточной степенью точности можно найти термодинамическим расчетом.

Сведения, касающиеся фазового равновесия «жидкость-пар» между оксидами азота и водными растворами азотной кислоты, относятся, главным образом, к гомогенным системам с низким парциальным давлением оксидов азота. В связи с этим представляет теоретический и практический интерес исследование равновесия «жидкость-пар» при повышенных парциальных давлениях оксидов азота в гетерогенной системе «NO- $\mathrm{NO}_{2}-\mathrm{HNO}_{3}-\mathrm{H}_{2} \mathrm{O}$ »

Так как при высоких парциальных давлениях оксидов азота существенное влияние на состав паровой фазы оказывают полимеризация $\mathrm{NO}_{2}$ и процесс образования $\mathrm{N}_{2} \mathrm{O}_{3}$, то важное значение приобретает правильный методологический подход к расчету равновесия. При расчете состава пара необходимо учитывать константу равновесия реакции полимеризации $\mathrm{NO}_{2}$, рассчитанную по уравнению Боденштейна [3], и константу равновесия реакции образования $\mathrm{N}_{2} \mathrm{O}_{3}$ [4].

Экспериментальная часть и обсуждение результатов

Основные исследования фазового равновесия «жидкость-пар» многокомпонентной расслаивающейся системы «оксиды азота-азотная кислота-вода» были проведены в диапазоне давлений 0,1-1,1 МПа при температурах 293-353 K, начальных массовых концентрациях $\mathrm{HNO}_{3} 40$ 68,4 мас.\% и исходных массовых соотношениях $\mathrm{N}_{2} \mathrm{O}_{4}: \mathrm{H}_{2} \mathrm{O}=1: 2 \ldots 12: 1$.

На основании полученных экспериментальных данных рассчитывали частные константы равновесия $\mathrm{K}_{1}$ и $\mathrm{K}_{2}$. При расчете парциальных давлений компонентов исходили из того, что их сумма равна общему давлению:

$$
\mathrm{P}_{\text {общ }}=\mathrm{P}_{\mathrm{NO}}+\mathrm{P}_{\mathrm{NO}_{2}}+\mathrm{P}_{\mathrm{N}_{2} \mathrm{O}_{4}}+\mathrm{P}_{\mathrm{N}_{2} \mathrm{O}_{3}}+\mathrm{P}_{\mathrm{HNO}_{3}}+\mathrm{P}_{\mathrm{H}_{2} \mathrm{O}} \text {. }
$$

Поскольку надежной методики непосредственного определения всех компонентов паровой фазы не существует, то их содержание выражали в пересчете на какой-либо из компонентов. Так, например, содержание $\mathrm{NO}$ и $\mathrm{N}_{2} \mathrm{O}_{3}$ выражено в пересчете на NO, а содержание $\mathrm{HNO}_{3}$ и $\mathrm{H}_{2} \mathrm{O}-$ в пересчете на $\mathrm{HNO}_{3}$. Составы жидкой и паровой фаз определяли аналитически в массовых процентах. Для раздельного определения каждого из компонентов паровой фазы и выражения массовых концентраций через мольные, применяли следующую методику. Массовая равновесная концентрация $\mathrm{HNO}_{3}$ в жидкой фазе определяется достаточно надежно. По ее значениям, используя имеющиеся в литературе сведения о парциальных давлениях $\mathrm{HNO}_{3}$ и $\mathrm{H}_{2} \mathrm{O}$ в парах при различных температурах и составах жидкой фазы, раздельно определяли мольные содержания азотной кислоты и воды в паровой фазе. Так как мольное содержание $\mathrm{HNO}_{3}$ и $\mathrm{H}_{2} \mathrm{O}$ в пересчете на азотную кислоту выражается уравнением:

$\mathrm{C}=\mathrm{C}_{\mathrm{HNO}_{3}}+7 \mathrm{C}_{\mathrm{H}_{2} \mathrm{O}}$,

а мольная концентрация $\mathrm{HNO}_{3}$ в пересчете на отбеленную кислоту, определяется по уравнению:

$$
\mathrm{C}_{\text {отб }}=\frac{\mathrm{C}_{\mathrm{HNO}_{3}}}{\mathrm{C}_{\mathrm{HNO}_{3}}+\mathrm{C}_{\mathrm{H}_{2} \mathrm{O}}} \cdot 100,
$$

находим, что:

$$
\begin{aligned}
& \mathrm{C}_{\mathrm{HNO}_{3}}=\mathrm{C} \frac{0,01 \mathrm{C}_{\text {отб }}}{7-0,06 \mathrm{C}_{\text {отб }}}, \\
& \mathrm{C}_{\mathrm{H}_{2} \mathrm{O}}=\mathrm{C} \frac{1-0,01 \mathrm{C}_{\text {отб }}}{7-0,06 \mathrm{C}_{\text {отб }}},
\end{aligned}
$$

где $\mathrm{C}_{\mathrm{HNO}_{3}}$ - содержание $\mathrm{HNO}_{3}$ в паровой фазе, мол.\%; $\mathrm{C}_{\mathrm{H}_{2} \mathrm{O}}-$ содержание $\mathrm{H}_{2} \mathrm{O}$ в паровой фазе, мол.\%; $\mathrm{C}-$ содержание $\mathrm{HNO}_{3}$ и $\mathrm{H}_{2} \mathrm{O}$ в пересчете на азотную кислоту, мол.\%; $\mathrm{C}_{\text {отб }}-$ содержание $\mathrm{HNO}_{3}$ в пересчете на отбеленную азотную кислоту, мол.\%.

Аналитически определив массовое суммарное содержание $\mathrm{NO}$ и $\mathrm{N}_{2} \mathrm{O}_{3}$ в пересчете на $\mathrm{NO}$ и приняв массовое содержание оксида азота(II) равным $\mathrm{C}_{\mathrm{No}}$, можно рассчитать массовое содержание $\mathrm{N}_{2} \mathrm{O}_{3}$ по уравнению: 


$$
\mathrm{C}_{\mathrm{N}_{2} \mathrm{O}_{3}}=\frac{76}{30}\left(\mathrm{C}_{\mathrm{NO}}^{*}-\mathrm{C}_{\mathrm{NO}}\right)
$$

где $\mathrm{C}_{\mathrm{N}_{2} \mathrm{O}_{3}}$ - содержание $\mathrm{N}_{2} \mathrm{O}_{3}$ в паровой фазе, мас.\%; $76^{3}$ и $30--$ молекулярные массы $\mathrm{N}_{2} \mathrm{O}_{3}$ и $\mathrm{NO}$, соответственно, кг/кмоль; $\mathrm{C}_{\mathrm{NO}}^{*}-$ суммарное содержание $\mathrm{NO}$ и $\mathrm{N}_{2} \mathrm{O}_{3}$ в пересчете на оKсид азота(II), мас.\%; $\mathrm{C}_{\mathrm{NO}}-$ содержание $\mathrm{NO}$ в паровой фазе, мас.\%.

Суммарное массовое содержание компонентов в паровой фазе составляет $100 \%$. Приняв массовое содержание $\mathrm{NO}_{2}$, равным $\mathrm{C}_{\mathrm{NO}_{2}}$, выражаем массовое содержание $\mathrm{N}_{2} \mathrm{O}_{4}$ в виде:

$$
\mathrm{C}_{\mathrm{N}_{2} \mathrm{O}_{4}}=\mathrm{B}-\mathrm{C}_{\mathrm{NO}_{2}}
$$

где $\mathrm{B}=100-\mathrm{C}_{\mathrm{NO}}^{*}-\mathrm{C}_{\mathrm{HNO}_{3}}-\mathrm{C}_{\mathrm{H}_{2} \mathrm{O}} ; \mathrm{C}_{\mathrm{HNO}_{3}}-$ coдержание $\mathrm{HNO}_{3}$ в паровой фазе, мас.\%; $\mathrm{C}_{\mathrm{H}_{2} \mathrm{O}}-$ содержание $\mathrm{H}_{2} \mathrm{O}$ в паровой фазе, мас.\%.

Мольная доля і-го компонента выражается через его массовое содержание уравнением:

$y_{i}=\frac{\frac{C_{i}}{M_{i}}}{\sum_{i=1}^{n} \frac{C_{i}}{M_{i}}}$,

где $\mathrm{y}_{\mathrm{i}}$ - мольная доля i-го компонента; $\mathrm{C}_{\mathrm{i}}$ - массовое содержание і-го компонента, кг; $\mathrm{M}_{\mathrm{i}}-$ молекулярная масса і-го компонента, кг/кмоль;

$\sum_{\mathrm{i}=1}^{\mathrm{n}} \frac{\mathrm{C}_{\mathrm{i}}}{\mathrm{M}_{\mathrm{i}}}-$ сумма количества вещества всех компонентов, входящих в состав паровой фазы, кмоль.

В данном случае общая сумма количества вещества всех компонентов (кмоль) может быть представлена в виде:

$$
\begin{aligned}
& \sum_{\mathrm{i}=1}^{\mathrm{n}} \frac{\mathrm{C}_{\mathrm{i}}}{\mathrm{M}_{\mathrm{i}}}=\frac{\mathrm{C}_{\mathrm{NO}}}{30}+\frac{\mathrm{C}_{\mathrm{N}_{2} \mathrm{O}_{3}}}{76}+\frac{\mathrm{C}_{\mathrm{N}_{2} \mathrm{O}_{4}}}{92}+ \\
& +\frac{\mathrm{C}_{\mathrm{NO}_{2}}}{46}+\frac{\mathrm{C}_{\mathrm{HNO}_{3}}}{63}+\frac{\mathrm{C}_{\mathrm{H}_{2} \mathrm{O}}}{18} .
\end{aligned}
$$

Подставив выражения (8) и (9) в уравнение (11), после преобразований получим:

$$
\sum_{\mathrm{i}=1}^{\mathrm{n}} \frac{\mathrm{C}_{\mathrm{i}}}{\mathrm{M}_{\mathrm{i}}}=\mathrm{A}+\frac{\mathrm{C}_{\mathrm{NO}_{2}}}{92} .
$$

где

$$
\begin{aligned}
\mathrm{A}= & \frac{62}{30 \cdot 92} \mathrm{C}_{\mathrm{NO}}^{*}+\frac{29}{63 \cdot 92} \mathrm{C}_{\mathrm{HNO}_{3}}+\frac{74}{18 \cdot 92} \mathrm{C}_{\mathrm{H}_{2} \mathrm{O}}+ \\
& +\frac{100}{92} .
\end{aligned}
$$

Из уравнения константы равновесия реакции полимеризации $\mathrm{NO}_{2}$ следует:

$\mathrm{P}_{\mathrm{NO}_{2}}^{2}-\mathrm{K}_{3} \cdot \mathrm{P}_{\mathrm{N}_{2} \mathrm{O}_{4}}=0$.

Согласно закону Дальтона:

$\mathrm{P}_{\mathrm{NO}_{2}}=\mathrm{y}_{\mathrm{NO}_{2}} \cdot \mathrm{P}_{\text {общ }}$,

$\mathrm{P}_{\mathrm{N}_{2} \mathrm{O}_{4}}=\mathrm{y}_{\mathrm{N}_{2} \mathrm{O}_{4}} \cdot \mathrm{P}_{\text {общ }}$.

Определив по уравнению (10) значения мольных долей $\mathrm{NO}_{2}$ и $\mathrm{N}_{2} \mathrm{O}_{4}$ и заменив по уравнению (12) $\sum_{\mathrm{i}=1}^{\mathrm{n}} \frac{\mathrm{C}_{\mathrm{i}}}{\mathrm{M}_{\mathrm{i}}}$ на $\mathrm{A}+\frac{\mathrm{C}_{\mathrm{NO}_{2}}}{92}$, по уравнениям (14) и (15) находим:

$\mathrm{P}_{\mathrm{NO}_{2}}=\frac{\mathrm{C}_{\mathrm{NO}_{2}}}{46 \cdot\left(\mathrm{A}+\frac{\mathrm{C}_{\mathrm{NO}_{2}}}{92}\right)} \mathrm{P}_{\text {общ }}$,

$\mathrm{P}_{\mathrm{N}_{2} \mathrm{O}_{4}}=\frac{\mathrm{C}_{\mathrm{N}_{2} \mathrm{O}_{4}}}{92 \cdot\left(\mathrm{A}+\frac{\mathrm{C}_{\mathrm{NO}_{2}}}{92}\right)} \mathrm{P}_{\text {обш }}$

Подставив выражения (16) и (17) в уравнение (13) и, заменив, согласно уравнению (9), $\mathrm{C}_{\mathrm{N}_{2} \mathrm{O}_{4}}$ на выражение $\mathrm{B}-\mathrm{C}_{\mathrm{NO}_{2}}$, после преобразования получим:

$$
\begin{aligned}
& \left(\mathrm{P}_{\text {общ }}+\frac{23}{92} \mathrm{~K}_{3}\right) \mathrm{C}_{\mathrm{NO}_{2}}^{2}+ \\
& +\left[\frac{23}{92} \mathrm{~K}_{3}(92 \mathrm{~A}-\mathrm{B})\right] \mathrm{C}_{\mathrm{NO}_{2}}-23 \mathrm{~K}_{3} \mathrm{AB}=0 .
\end{aligned}
$$


По уравнению (18) определяем массовое содержание $\mathrm{NO}_{2}$, по уравнению (9) - массовое содержание $\mathrm{N}_{2} \mathrm{O}_{4}$ и по уравнению (12) - сумму количества вещества (кмоль) компонентов паровой фазы.

Из уравнения константы равновесия реакции образования $\mathrm{N}_{2} \mathrm{O}_{3}$ рассчитываем массовое содержание $\mathrm{NO}$ и $\mathrm{N}_{2} \mathrm{O}_{3}$. Парциальные давления $\mathrm{NO}$ и $\mathrm{N}_{2} \mathrm{O}_{3}$ равны:

$$
\begin{aligned}
& \mathrm{P}_{\mathrm{NO}}=\mathrm{y}_{\mathrm{NO}} \mathrm{P}_{\text {общ }}=\frac{\mathrm{C}_{\mathrm{NO}}}{30 \cdot \sum_{\mathrm{i}=1}^{\mathrm{n}} \frac{\mathrm{C}_{\mathrm{i}}}{\mathrm{M}_{\mathrm{i}}}} \mathrm{P}_{\text {общ }}, \\
& \mathrm{P}_{\mathrm{N}_{2} \mathrm{O}_{3}}=\mathrm{y}_{\mathrm{N}_{2} \mathrm{O}_{3}} \mathrm{P}_{\text {общ }}=\frac{\mathrm{C}_{\mathrm{N}_{2} \mathrm{O}_{3}}}{76 \cdot \sum_{\mathrm{i}=1}^{\mathrm{n}} \frac{\mathrm{C}_{\mathrm{i}}}{\mathrm{M}_{\mathrm{i}}}} \mathrm{P}_{\text {общ }} .
\end{aligned}
$$

Подставив значения парциальных давлений $\mathrm{NO}_{2}, \mathrm{NO}, \mathrm{N}_{2} \mathrm{O}_{3}$ соответственно из уравнений (16), (19), (20) в выражение для константы равновесия реакции образования $\mathrm{N}_{2} \mathrm{O}_{3}$ :

$$
\mathrm{K}_{4}=\frac{\mathrm{P}_{\mathrm{NO}} \mathrm{P}_{\mathrm{NO}_{2}}}{\mathrm{P}_{\mathrm{N}_{2} \mathrm{O}_{3}}},
$$

после преобразования получаем:

$$
\mathrm{C}_{\mathrm{NO}}=\frac{\mathrm{C}_{\mathrm{NO}}^{*}}{1+\frac{\mathrm{C}_{\mathrm{NO}_{2}}}{46 \mathrm{~K}_{4} \sum_{\mathrm{i}=1}^{\mathrm{n}} \frac{\mathrm{C}_{\mathrm{i}}}{\mathrm{M}_{\mathrm{i}}}} \mathrm{P}_{\text {общ }}} .
$$

Содержание $\mathrm{N}_{2} \mathrm{O}_{3}$ рассчитываем по уравнению (8).

По значениям парциальных давлений NO и $\mathrm{NO}_{2}$ рассчитывали частную константу равновесия $\mathrm{K}_{1}$. Рассчитанные по результатам экспериментов и по предложенной методике значения $\lg \mathrm{K}_{1}$, представленные на рисунке, показывают, что для исследованной области массовых соотношений $\mathrm{N}_{2} \mathrm{O}_{4}: \mathrm{H}_{2} \mathrm{O}$, (равновесная массовая концентрация $\mathrm{HNO}_{3} 52,0-75,8 \%$ ), при любых начальных концентрациях азотной кислоты зависимость $\lg \mathrm{K}_{1}$ от равновесной концентрации $\mathrm{HNO}_{3}$ имеет линейный характер и согласуется с литературными данными [1,2]. Полученные данные свидетельствуют о том, что частная константа равновесия $\mathrm{K}_{1}$ не зависит от давления в системе.

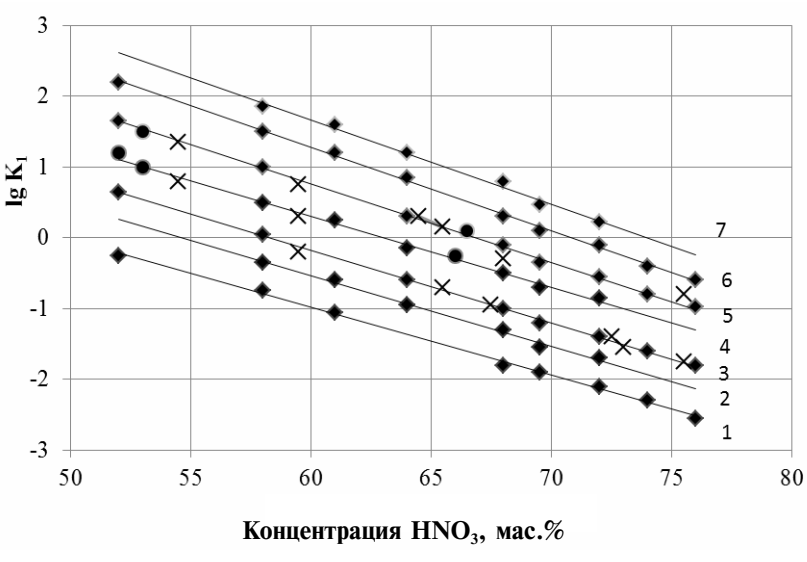

Зависимость $\lg \mathrm{K}_{1}$ от равновесной концентрации $\mathrm{HNO}_{3}$ при различных температурах, K: $1-353,2-343,3-333$, $4-323,5-313,6-303,7-293$. - - полученные данные; - данные работы [1]; $\mathbf{x}$ - данные работы [2]

Частная константа равновесия $\mathrm{K}_{1}$ может быть определена через общую константу равновесия К. Величину частной константы равновесия $\mathrm{K}_{2}$ определяют по имеющимся в литературе данным о составе паров над растворами азотной кислоты, а общую константу равновесия $\mathrm{K}_{298}$ при стандартных условиях рассчитывают по уравнению:

$\lg \mathrm{K}_{298}=-\frac{\Delta \mathrm{G}_{298}^{0}}{2,303 \mathrm{R} \cdot 298}$,

где $\Delta \mathrm{G}_{298}^{0}-$ изменение свободной энергии при стандартных условиях, Дж/моль;

$\mathrm{R}=8,314$ Дж/(моль-град) - универсальная газовая постоянная; 298 - стандартная температуpa, K.

При других температурах общую константу равновесия $\mathrm{K}_{\mathrm{T}}$ определяли по уравнению:

$\lg \mathrm{K}_{\mathrm{T}}=\lg \mathrm{K}_{298}+\frac{\Delta \mathrm{H}_{298}^{0}}{2,303 \mathrm{R} \cdot 298}-\frac{\Delta \mathrm{H}_{298}^{0}}{2,303 \mathrm{RT}}$,

где $\Delta \mathrm{H}_{298}^{0}-$ изменение стандартной энтальпии образования, Дж/моль; Т - температура, К.

Расчет состава паровой фазы через общую константу равновесия позволяет значительно упростить расчет равновесий. Однако не было ясно, правомерен ли такой расчет равновесия для расслаивающихся систем.

С целью проверки сопоставимости экспериментальных и расчетных данных разработан метод определения парциальных давлений 
компонентов паровой фазы через константы равновесия, вычисленные по термодинамическим функциям. Из уравнений частной константы равновесия $\mathrm{K}_{1}$, константы равновесия реакции полимеризации диоксида азота $\mathrm{K}_{3}$ и константы равновесия $\mathrm{K}_{4}$ реакции образования оксида азота(III) имеем:

$$
\begin{aligned}
& \mathrm{P}_{\mathrm{NO}}=\mathrm{K}_{1} \mathrm{P}_{\mathrm{NO}_{2}}^{3}, \\
& \mathrm{P}_{\mathrm{N}_{2} \mathrm{O}_{4}}=\frac{1}{\mathrm{~K}_{3}} \mathrm{P}_{\mathrm{NO}_{2}}^{2}, \\
& \mathrm{P}_{\mathrm{N}_{2} \mathrm{O}_{3}}=\frac{\mathrm{K}_{1}}{\mathrm{~K}_{4}} \mathrm{P}_{\mathrm{NO}_{2}}^{4} .
\end{aligned}
$$

Подставив значения парциальных давлений $\mathrm{NO}, \mathrm{N}_{2} \mathrm{O}_{4}$ и $\mathrm{N}_{2} \mathrm{O}_{3}$ из уравнений (25), (26) и (27) в выражение (3), получим:

$$
\begin{aligned}
& \frac{\mathrm{K}_{1}}{\mathrm{~K}_{4}} \mathrm{P}_{\mathrm{NO}_{2}}^{4}+\mathrm{K}_{1} \mathrm{P}_{\mathrm{NO}_{2}}^{3}+\frac{1}{\mathrm{~K}_{3}} \mathrm{P}_{\mathrm{NO}_{2}}^{2}+\mathrm{P}_{\mathrm{NO}_{2}}+ \\
& +\mathrm{P}_{\mathrm{HNO}_{3}}+\mathrm{P}_{\mathrm{H}_{2} \mathrm{O}}-\mathrm{P}_{\text {общ }}=0 .
\end{aligned}
$$

Парциальные давления азотной кислоты и воды определяют по имеющимся в литературе данным. По уравнению (28) рассчитываем парциальное давление $\mathrm{NO}_{2}$ и далее по уравнениям (25), (26) и (27) находим соответственно парциальные давления $\mathrm{NO}, \mathrm{N}_{2} \mathrm{O}_{4}$ и $\mathrm{N}_{2} \mathrm{O}_{3}$. Зная парциальные давления компонентов паровой фазы, рассчитываем их массовые содержания. Концентрации $\mathrm{NO}$ и $\mathrm{N}_{2} \mathrm{O}_{3}$ выражали в пересчете на $\mathrm{NO}$, а концентрации $\mathrm{HNO}_{3}$ и $\mathrm{H}_{2} \mathrm{O}-$ в пересчете на $\mathrm{HNO}_{3}$. Сравнив экспериментальные данные с данными, рассчитанными по термодинамическим функциям, было установлено, что они удовлетворительно согласуются. Относительная ошибка между экспериментом и расчетом составила около $5 \%$.

Следовательно, применение термодинамического расчета для определения состава паровой фазы в расслаивающейся системе «оксиды азота-азотная кислота-вода» свидетельствует о том, что он является таким же правомерным, как и для расчета аналогичных гомогенных систем.

\section{Выводы}

Таким образом, установлено влияние состава жидкой фазы расслаивающихся смесей «оксиды азота-азотная кислота-вода» на состав паров и показано, что изменение концентраций компонентов в паровой фазе определяется равновесной концентрацией азотной кислоты в жидкости и температурой.

\section{СПИСОК ЛИТЕРАТУРЫ}

1. Куча М.И., Зубов В.В. О равновесном составе окислов азота в системе окислы азота (газ) $-\mathrm{HNO}_{3}-\mathrm{H}_{2} \mathrm{O}-$ окислы азота (жидкость) // Труды ЛИТЛ. - 1976. - № 17. C.19-24.

2. Равновесие оксидов азота с растворами азотной кислоты / Терещенко Л.Я., Куча М.И., Панов В.П., Зубов В.В. // Журн. прикл. хим. - 1979. - Т.52. - № 3. - С.1743-1747.

3. Bodenstein M. Bildung und Zerlegung von Stickoxid // Z. Physik. Chem. -1922. - Vol.100. - P.63-72.

4. Verhock F., Daniels F. The dissociation constants of nitrogen tetroxide and of nitrogen trioxide // J. Am. Chem. Soc. - 1931. - Vol.53. - P.1250-1263.

5. Десорбция оксидов азота из продукционной азотной кислоты (58-60\% масс.) / Литвиненко А.А., Печенко Т.И., Подустов М.А., Букатенко А.И. // Восточно-Европ. журн. передовых технол. - 2015. - № 5/6 (77). - С.43-48.

6. Kuporka J. Removal of nitrogen oxides from flue gases in a packed column // Environ. Prot. Eng. - 2011. - Vol.37. No. 1. - P.13-22.

7. Intensification of nitrous acid oxidation / Chacuk A., Miller J.S., Wilk M., Ledakowicz S // Chem. Eng. Sci. - 2007. - Vol. 62, Issue 24. - P. 7446-7453.

8. De Paiva J.L., Kachan G.C. Absorption of nitrogen oxides in aqueous solutions in a structured packing pilot column // Chem. Eng. Process. - 2004. - Vol.43. - No. 7. - P.941-948.

9. Skalska, K., J.S. Miller J.S., Ledakowicz S. Kinetic model of $\mathrm{NO}_{\mathrm{x}}$ ozonation and its experimental verification // Chem. Eng. Sci. - 2011. - Vol.66. - No. 14. - P.3386-3391.

10. Janiczek $W$. A simplified model of the absorptiveregenerative process in the technology of nitric acid production // Techn. Trans. Chem. - 2014. - Vol.1. - P.15-33.

11. Сравнительная оценка и поиск новых эффективных технологических схем очистки газовоздушных выбросов от оксидов азота $\left(\mathrm{NO}_{\mathrm{x}}\right)$ / Письменный Б.В., Кравченко Л.П., Деримова А.В., Кожура О.В. // Вопр. химии и хим. технол. - 2011. - № 6. - С.173-176.

12. Термодинамические расчеты реакций, протекающих при получении растворов $\mathrm{N}_{2} \mathrm{O}_{3}-\mathrm{N}_{2} \mathrm{O}_{4}$ / Созонтов В.И., Суворин А.В., Казаков В.В., Кошовец Н.В., Ожередова М.А // Вісник СНУ ім. В. Даля. - 2017. - № 5 (235). - С.16-19. 


\section{ДОСЛІДЖЕННЯ СКЛАДУ ПАРОВОЇ ФАЗИ НАД ВОДНИМИ РОЗЧИНАМИ НІТРАТНОЇ КИСЛОТИ ТА ОКСИДІВ АЗОТУ, ЩО РОЗШАРОВУЮТЬСЯ}

\section{В.Г. Созонтов, О.Г. Архипов, О.В. Суворін В.М., Москалик, І.Л. Коваленко}

У роботі теоретично обтрунтовані і експериментально вивчені закономірності фазової рівноваги рідина-пара у багатокомпонентній системі «оксиди азоту-нітратна кислотавода» під тиском 0,1-1,1 МПа, що розшаровується. Розроблена методика розрахунку складу парової фази, яка передбачає процеси полімеризації діоксиду азоту і утворення нітроген(III) оксиду. За значеннями парціальних тисків $\mathrm{NO}$ i $\mathrm{NO}_{2}$ розрахована частинна константа рівноваги реакції кислотоутворення і показано, що для дослідженого діапазону масових співвідношень $\mathrm{N}_{2} \mathrm{O}_{4}: \mathrm{H}_{2} \mathrm{O}=1: 2 \ldots 12: 1$ при початкових концентраціях нітратної кислоти 40,0-68,4 мас.\% залежність логарифму частинної константи рівноваги від рівноважної концентраціі $\mathrm{HNO}_{3}$ має лінійний характер, що свідчить про незалежність частинної константи рівноваги від тиску. Встановлено, шо частинна константа рівноваги може бути визначена через загальну константу рівноваги, розраховану за термодинамічними константами, і частинну константу рівноваги, знайдену за париіальними тисками $\mathrm{HNO}_{3} \mathrm{i}_{2} \mathrm{O}$. Розрахований склад парової фази і показано, що експериментальні дані задовільно узгоджуються з даними, отриманими за термодинамічними функціями. Відносна похибка між експериментальними і розрахунковими даними склала близько $5 \%$.

Ключові слова: оксиди азоту, нітратна кислота, рівноважний склад, парціальний тиск, частинна константа рівноваги.

\section{THE STUDY OF THE COMPOSITION OF THE VAPOR PHASE ABOVE THE STRATIFIED AQUEOUS SOLUTIONS OF NITRIC ACID AND NITROGEN OXIDES}

V.G. Sozontov ${ }^{a}$, , O.G. Arkhypov ${ }^{a}$, O.V. Suvorin ${ }^{a}$, V.M. Moskalyk ${ }^{a}$, I.L. Kovalenko ${ }^{b}$

a Volodymyr Dahl East Ukrainian National University, Severodonetsk, Ukraine

b Ukrainian State University of Chemical Technology, Dnipro, Ukraine

* e-mail: moskalyk2017@gmail.com

The present work considers the main characteristics of phase equilibrium «liquid-vapor» in a multicomponent stratified system «nitrogen oxides-nitric acid-water» at a pressure of $0.1-1.1 \mathrm{MPa}$. The method is developed which allows calculating the composition of vapor phase and involves the polymerization of nitrogen dioxide and the formation of dinitrogen trioxide. Using the values of partial pressures of $\mathrm{NO}$ and $\mathrm{NO}_{2}$, the particular equilibrium constant of the reaction of acid formation is calculated. It is shown that the dependence of the logarithm of particular equilibrium constant on the equilibrium $\mathrm{HNO}_{3}$ concentration is linear at the studied mass ratio $\mathrm{N}_{2} \mathrm{O}_{4}: \mathrm{H}_{2} \mathrm{O}=1: 2 . .12: 1$ and the initial concentration of nitric acid of 40.0-68.4 wt.\%. This indicates that particular equilibrium constant does not depend on the pressure. It is established that the particular equilibrium constant can be determined taking the overall equilibrium constant calculated via thermodynamic constants and the particular equilibrium constant calculated from the partial pressures of $\mathrm{HNO}_{3}$ and $\mathrm{H}_{2} \mathrm{O}$. The composition of the vapor phase is calculated and the experimental data are shown to satisfactorily agree with the data obtained for the thermodynamic functions. $A$ relative error between experimental and calculated data is about $5 \%$.
Keywords: nitrogen oxides; nitric acid; equilibrium composition; partial pressure; particular equilibrium constant.

\section{REFERENCES}

1. Kucha M.I., Zubov V.V. O ravnovesnom sostave okislov azota $v$ sisteme okisly azota $(\mathrm{gaz})-\mathrm{HNO}_{3}-\mathrm{H}_{2} \mathrm{O}-$ okisly azota (zhidkost') [On the equilibrium composition of nitrogen oxides in the system of oxides of nitrogen (gas) $-\mathrm{HNO}_{3}-\mathrm{H}_{2} \mathrm{O}-$ oxides of nitrogen (liquid)]. Trudy LITL, 1976, no. 17, pp. 19-24. (in Russian).

2. Tereschenko L.Ya., Kucha M.I., Panov V.P., Zubov V.V. Ravnovesie oksidov azota s rastvorami azotnoi kisloty [Equilibrium of nitrogen oxides with solutions of nitric acid]. Zhurnal Prikladnoi Khimii, 1979, vol. 52, no. 3, pp. 1743-1747. (in Russian).

3. Bodenstein M. Bildung und Zerlegung von Stickoxid. Zeitschrift für Physikalische Chemie, 1922, vol. 100, pp. 63-72. (in German).

4. Verhock F.H., Daniels Farrington. The dissociation constants of nitrogen tetroxide and of nitrogen trioxide. Journal of the American Chemical Society, 1931, vol. 53, pp. 1250-1263.

5. Litvinenko A.A., Pechenko T.I., Podustov M.A., Bukatenko A.I. Desorbtsiya oksidov azota iz produktsionnoi azotnoi kisloty (58-60 mass.\%) [Desorption of nitrogen oxides from production of nitric acid (58-60 wt.\%)]. Eastern-European Journal of Enterprise Technologies, 2015, no. 5/6 (77), pp. 43-48. (in Russian).

6. Kuporka J. Removal of nitrogen oxides from flue gases in a packed column. Environment Protection Engineering, 2011, vol. 37 , no. 1 , pp. 13-22.

7. Chacuk A., Miller J.S., Wilk M., Ledakowicz S. Intensification of nitrous acid oxidation. Chemical Engineering Science, 2007, vol. 62, pp. 7446-7453.

8. De Paiva J.L., Kachan G.C. Absorption of nitrogen oxides in aqueous solutions in a structured packing pilot column. Chemical Engineering and Processing: Process Intensification, 2004, vol. 43 , pp. $941-948$

9. Skalska K., Miller J.S., Ledakowicz S. Kinetic model of $\mathrm{NO}_{x}$ ozonation and its experimental verification. Chemical Engineering Science, 2011, vol. 66, pp. 3386-3391.

10. Janiczek W. A simplified model of the absorptiveregenerative process in the technology of nitric acid production. Technical Transactions. Chemistry, 2014, vol. 1, pp. 15-33.

11. Pismennyiy B.V., Kravchenko L.P., Derimova A.V., Kozhura O.A. Sravnitel'naya otsenka i poisk novykh effektivnykh tekhnologicheskikh skhem ochistki gazovozdushnykh vybrosov ot oksidov azota $\left(\mathrm{NO}_{\mathrm{x}}\right)$ [Comparative assessment and search of new efficient manufacturing schemes of the purification of airgas emissions from nitrogen oxides $\left.\left(\mathrm{NO}_{\mathrm{x}}\right)\right]$. Voprosy Khimii $i$ Khimicheskoi Tekhnologii, 2011, no. 6, pp. 173-176. (in Russian).

12. Sozontov V.I., Suvorin A.V., Kazakov V.V., Koshovets N.V., Ozheredova M.A. Termodinamicheskie raschety reaktsii, protekayuschikh pri poluchenii rastvorov $\mathrm{N}_{2} \mathrm{O}_{3}-\mathrm{N}_{2} \mathrm{O}_{4}$ [Thermodynamic calculations of reactions occurring upon receipt of the solutions $\mathrm{N}_{2} \mathrm{O}_{3}-\mathrm{N}_{2} \mathrm{O}_{4}$. Bulletin of the Volodymyr Dahl East Ukrainian National University, 2017, no. 5 (235), pp. 16-19. (in Russian). 